\section{UV Absorbance Measurements of DNA in Microplates}

\section{BioTechniques 28:60-64 (January 2000)}

UV absorbance measurements in microplates became possible only five years ago when Molecular Devices (Sunnyvale, CA, USA) introduced the SPECTRAmax ${ }^{\circledR} 250$, the first UV-Visible microplate spectrophotometer. Microplates have since become very popular for making UV measurements of DNA, RNA and protein concentrations because of their higher throughput compared to traditional cuvettes. Despite their huge popularity, many people experience difficulty when adapting assays to them.

Especially in the UV range, microplate assays require an awareness of the optical properties of the microplate materials and more attention to technique than do traditional cuvettes to get accurate absorbance results. Absorbance measurements made through microplates are subject to path length variability and are vulnerable to interference from surface effects at the air/liquid interface. Samples have different absorbance values when read in microplates and cuvettes, unless the microplate values are normalized for optical path length. Finally, modern microplate spectrophotometers are designed with increasingly narrow beams to accommodate smaller microplate wells and thus are much more vulnerable to spurious readings caused by particles than older, wide-beam instruments. Lack of attention to these details is the most frequent cause of difficulty in adapting assays to microplates.

The following report provides guidelines for optimizing DNA measurements in microplates. It also gives an example of expected results if the guidelines are followed and illustrates the effect of ionic strength on DNA absorptivity.

UV Transparent Microplates. Quartz microplates are commercially available (Hellma Cells, Forest Hills, NY, USA), but are expensive (>\$1000). Standard polystyrene microplates do not transmit light below approximately $300 \mathrm{~nm}$. However, there are at least three UV transparent plastic microplates available (Figure 1). The UVPlate $^{\mathrm{TM}}$ (Corning-Costar, Cambridge, MA, USA) and the UV-Star ${ }^{\circledR}$ (Greiner, available from E \& K Scientific Products, Saratoga, CA, USA) have good transmission down to approximately $215 \mathrm{~nm}$. The UVMax ${ }^{\mathrm{TM}}$ (Polyfiltronics, available from Phenix Research Products, Hayward, CA, USA) is usable down to $240 \mathrm{~nm}$. The UV-Plate and UV-Star are comparable to a quartz microplate for DNA/RNA analyses. UVMax microplates have a higher background, but good results can be obtained by prereading the plates and subtracting the background on a wellby-well basis.

Recommendations for optimizing DNA absorbance measurements $(3,4)$. First, use clean microplates and particlefree solutions. Particles can cause artifactual spikes in absorbance values by as many as 0.3 absorbance units in modern narrow-beam instruments. Remove particles by filtering buffers (e.g., pore size $\leq 5 \mu$ ). If needed, remove dust by blowing out the microplate with clean dry air. Cover microplate if it is not to be read immediately.

Second, note absorbance values of buffer blanks (and include blanks at least in duplicate). The UV-Plate and UV-Star microplates vary slightly between lots, but the mean $\mathrm{A}_{260}$ value for a water-filled plate should be between 0.046 and 0.060 with a SD $\leq 0.002$. The mean $A_{260}$ value for a water-filled quartz plate should be $0.03-0.04$ with a $\mathrm{SD} \leq 0.002$. Buffer-containing blanks may have higher values. If the blank values do not fall into the expected range, the most likely cause is a dirty microplate, particles in the wells or a defective microplate.

Third, if your procedure calls for a small sample volume $(1-20 \mu \mathrm{L})$ and a diluent, pipet the small volume into the well first, followed by the larger volume. Mix well with the pipettor or by vibrating the microplate.

Fourth, recognize that absorbance depends on the meniscus curvature as well as the sample volume. The optical path length (and therefore the absorbance) of a microplate sample depends not only on its volume but also on the degree of meniscus curvature. The meniscus can be nearly flat (water or buffer in untreated microplates) or highly curved (detergent-containing sample or surface-treated microplate). The resulting difference

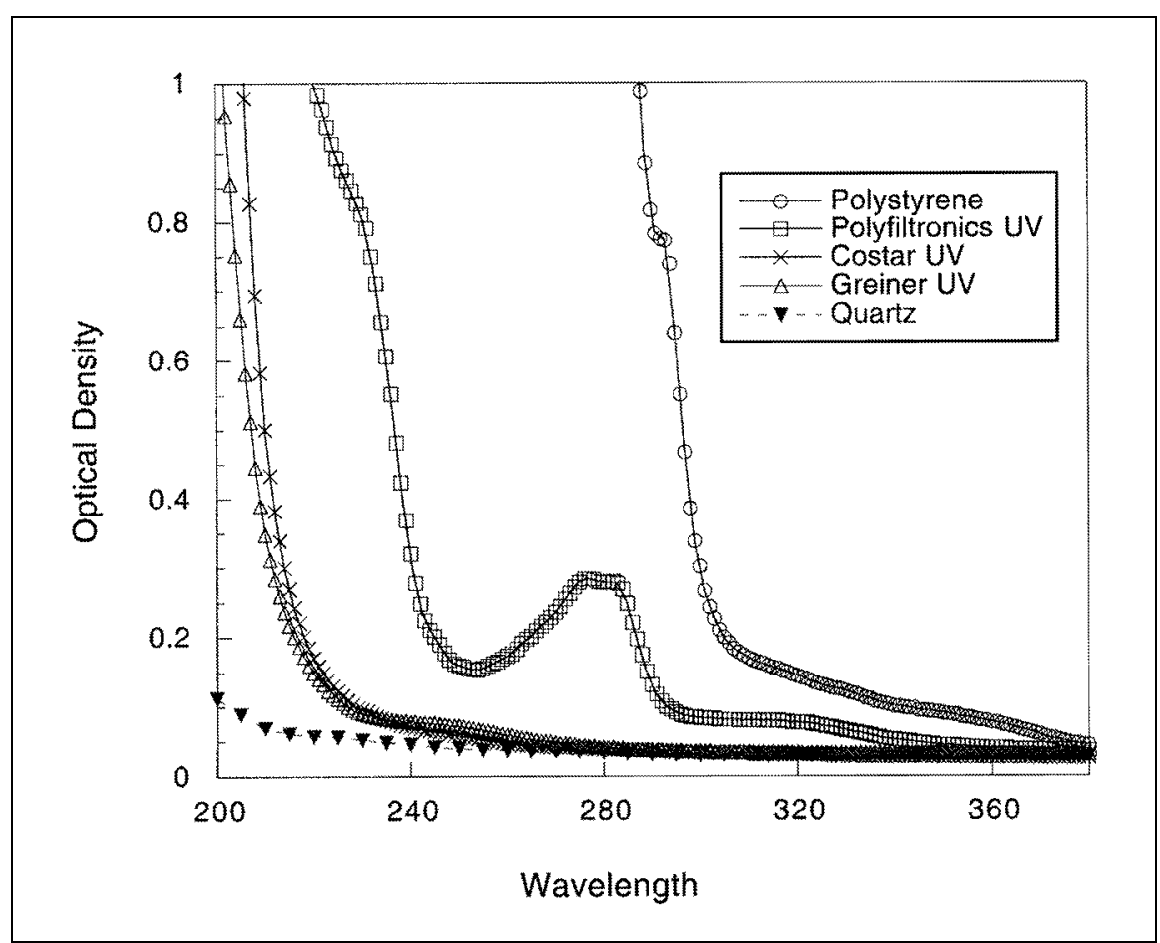

Figure 1. Absorbance spectra of microplates (containing $200 \mu \mathrm{L}$ water per well) in a SPECTRAmax PLUS. Left to right: Quartz, UV-Star, UV-Plate, UVMax and generic polystyrene. 
in optical path length can be as much as $25 \%$ for $100 \mu \mathrm{L}$ samples. Depending on the solution composition, the meniscus curvature in DNA samples can range between the two extremes.

Fifth, be aware that increased ionic strength decreases absorbance $(1,6)$. DNA has approximately $23 \%$ lower absorbance when dissolved in buffered saline rather than water.

Sixth, for low absorbing samples, use the maximum possible volume (250$300 \mu \mathrm{L}$ in a standard 96-well plate) to get maximum optical path length. Include duplicates or triplicates if possible.

Example of DNA measurement in microplates. If the guidelines given above are heeded, $\mathrm{A}_{260}$ measurements of DNA in microplates are comparable to those obtained in a conventional spectrophotometer. In a standard 96well microplate, a full well $(300 \mu \mathrm{L})$ has an optical path length of nearly 1 $\mathrm{cm}$. Figure 2 shows an example of a low-level DNA standard curve obtained in a microplate. The calculated limit of detection (amount producing an absorbance value higher than 3 positive SDs of the blank values above zero) was approximately $26 \mathrm{ng} /$ well. The calculated limit of quantitation was approximately $75 \mathrm{ng} /$ well, using a very conservative criterion (amount producing an absorbance value higher than 10 positive SDs of the blank above zero). This limit translates to $250 \mathrm{ng} / \mathrm{mL}$ for a sample volume of $300 \mu \mathrm{L}$. The microplate results agree favorably with published limits of detection of DNA using $\mathrm{A}_{260}$ measurements $(2,5)$. Quantitation of lower DNA concentrations requires an alternative technique, such as use of a fluorescent dye.

DNA estimation using absorptivity. DNA concentration is commonly estimated by dividing the $\mathrm{A}_{260}$ value by

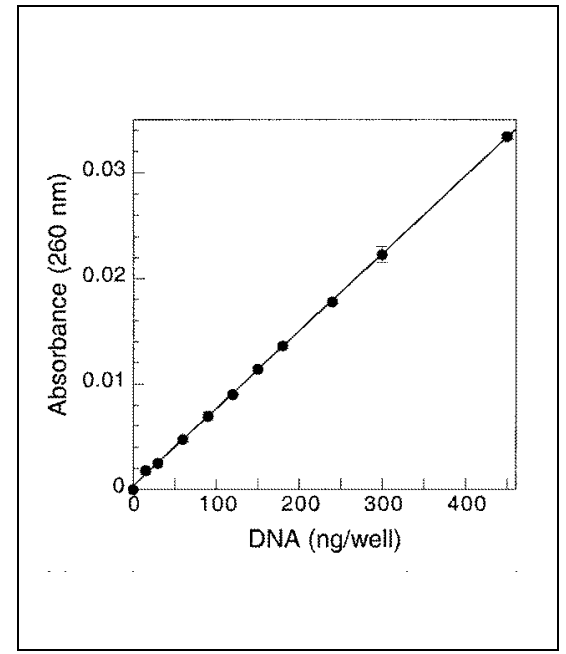

Figure 2. Standard curve obtained with calf thymus DNA (Type I; Sigma, St. Louis, MO, USA) dissolved in water and diluted to concentrations ranging from $0.1-1.5 \mu \mathrm{g} / \mathrm{mL}$. Triplicate aliquots of $300 \mu \mathrm{L} /$ well were read in a UVPlate. Absorbance measurements were made in a SPECTRAmax PLUS. 
the $1 \mathrm{~cm}$ absorptivity value (or multiplying by its reciprocal). To use that value for microplate samples, absorbance values must be normalized to a $1 \mathrm{~cm}$ path length. This can be done by determining a conversion factor for a given sample volume and microplate. Alternatively, several microplate spectrophotometers use infrared absorbance measurements to determine path length on a well-by-well basis, and absorbance values can be normalized automatically.

The $\mathrm{A}^{-1}$ value for double-stranded DNA at $260 \mathrm{~nm}$ is commonly approximated as $50 \mathrm{~cm} \mu \mathrm{g} / \mathrm{mL}$ for a $1 \mathrm{~cm}$ path length $(2,5)$. The dependence of nucleic acid absorbance on ionic strength has been reported previously $(1,6)$, but is not generally recognized. Figure 3 illustrates the difference in absorbance of DNA prepared in water, Tris (TE) buffer and Tris-buffered saline (TES). $\mathrm{A}_{260}$ values were approximately $15 \%$ lower in TE and 23\% lower in TES when compared to the same concentrations in water. The calculated $\mathrm{A}^{-1}$ values are 38,45 and $50 \mathrm{~cm} \mu \mathrm{g} / \mathrm{mL}$ for DNA in water, TE and TES, respectively (Figure 3). Thus, the commonly used value of 50 is correct only for relatively high ionic strength solutions. A lower detection limit for DNA by $\mathrm{A}_{260}$ measurement may be achieved if the samples are dissolved in deionized water rather than a salt solution.

With attention to technique, accurate and reproducible DNA measurements

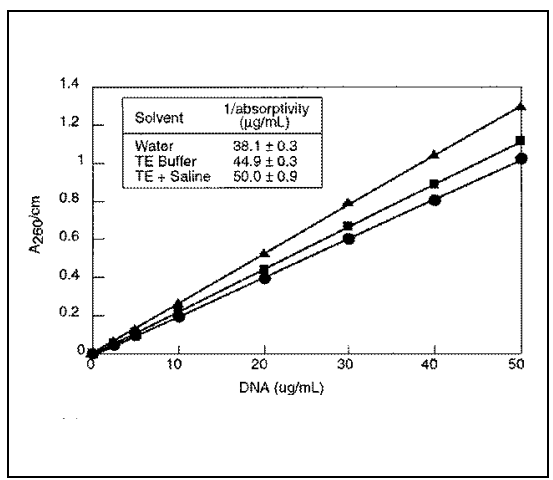

Figure 3. Effect of ionic strength on absorbance of DNA at $260 \mathrm{~nm}$. DNA (Type I; Sigma) was dissolved in deionized water (top curve), TE buffer; $10 \mathrm{mM}$ Tris, $1 \mathrm{mM}$ EDTA, pH 7.4 (middle plot), or TES buffer (TE buffer $+0.9 \%$ $\mathrm{NaCl}$ ) (bottom curve). Absorbance measurements were made in a SPECTRAmax PLUS. Inset: $\mathrm{A}^{-1}$ values; average of 7 concentrations $(2.5-50 \mu \mathrm{g} / \mathrm{mL}), 4$ replicates each. can be made easily in microplates. The lower limits of detection and quantitation are comparable to those obtained with semi-micro cuvettes in conventional spectrophotometers.

\section{REFERENCES}

1.Beaven, G.H., E.R. Holiday and E.A. Johnson. 1955. Optical properties of nucleic acids and their components, pp. 493-553. In E. Chargaff and J.N. Davidson (Eds). The Nucleic Acids, Vol 1. Academic Press, New York.

2.Gallagher, S.R. 1994. Quantitation of DNA and RNA with absorption and fluorescence spectroscopy. p. A3.D.1-A3.D3. In Current Protocols In Molecular Biology, Vol 3. John Wiley \& Sons, New York.

3.McGown, E.L. 1999. DNA and RNA Measurements in SPECTRAmax ${ }^{\circledR}$ Microplate Spectrophotometers. Maxline Application Note No. 33. Molecular Devices, Sunnyvale, CA.

4.McGown, E.L. 1999. UV Measurements in Microplates. Maxline Application Note No. 32. Molecular Devices, Sunnyvale, CA.

5.Sambrook, J., E.F. Fritsch and T. Maniatis. 1989. Molecular Cloning: A Laboratory Manual, 2nd ed. p. E.5. CSH Laboratory Press, Cold Spring Harbor, New York.

6.Wilfinger, W.W., K. Mackey and P. Chomczynski. 1997. Effect of $\mathrm{pH}$ and ionic strength on the spectrophotometric assessment of nucleic acid purity. BioTechniques 22:474-479.

Address correspondence to Dr. Evelyn L. McGown, Senior Applications Specialist Molecular Devices Corporation, Sunnyvale, CA 94089-1136, USA. Internet: evelyn_mc gown@moldev.com

Received 21 September 1999; accepted 12 October 1999.

\section{Evelyn L. McGown Molecular Devices Corporation Sunnyvale, CA, USA}

\section{Improved Purification of the Double-Stranded RNA from Killer Strains of Yeast}

BioTechniques 28:64-65 (January 2000)

The importance of studying yeast killer strains becomes apparent when one considers the mechanisms involved in the life cycle of double-stranded RNA viruses. Interests ranging from the analysis of viral machinery to the complex interaction of the host's relationship with the killer virus makes the system an attractive source of information. Additionally, for those studying toxin production, secretion and mode of action, which can be connected by analogy to hormone chemistry, the yeast killer strains serve as an excellent model system (3-5). The use of these self-selecting, extrachromosomal genetic elements as a new vector system could also lead to a drastic simplification of existing target gene purification techniques (5). Protein processing in eukaryotes and the connection often involved with higher eukaryotes, such as posttranslational modification of gene products, also leads to an intense interest in the study of model systems such as the yeast killer phenomenon.

Researchers currently working with the dsRNA virus found in yeast killer strains often use a modified version of the Fried and Fink protocol (1) for extracting total nucleic acids. The rather tedious and time-consuming protocol involves numerous centrifugations, incubations and phenol extractions. For this study, the following version of the Fried and Fink protocol was used: pelleted cells were resuspended in $50 \mathrm{mM}$ EDTA, vortex mixed, pelleted and resuspended in $50 \mathrm{mM}$ Tris- $\mathrm{H}_{2} \mathrm{SO}_{4}(\mathrm{pH}$ 9.3) with $2.5 \% \beta$-mercaptoethanol, incubated $15 \mathrm{~min}$ and pelleted. To the pellet, lysis buffer $(0.1 \mathrm{M} \mathrm{NaCl}, 10 \mathrm{mM}$ Tris-HCl, pH 7.5, 10 mM EDTA, 0.2\% SDS) and an equal volume of phenol were added and the mixture was incubated on a rocker platform for $1 \mathrm{~h}$. The mixture was centrifuged at $12000 \times g$ for $5 \mathrm{~min}$ and the aqueous phase (top layer) was collected and extracted again with phenol/chloroform. The 